\title{
Significant IOP reduction after combined phacoemulsification and micro-invasive glaucoma surgery (MIGS)
}

\author{
Ulrich-Peter Best* and Harry Domack \\ Eye Clinic and Laser Eye Clinic Mainfranken, Am Oberen Marienbach 1, D-97421 Schweinfurt, Germany
}

\begin{abstract}
In a controlled clinical trial, we investigated long-term results after trabecular micro-bypass stent implantation in routine cataract surgery in eyes with chronic open angle glaucoma. Combined phacoemulsification and trabecular micro-bypass implantation proved to be an effective and safe treatment method to reduce intraocular pressure, reducing the burden on local drug treatment. Implantation offers a good option for additional pressure reduction. Microsurgical procedures to reduce intraocular pressure, which are minimally traumatic and are performed ab interno and characterized by efficiency, a high safety profile and a rapid healing process, should be used more frequently.
\end{abstract}

\section{Introduction}

Since decades, trabeculectomy is the gold standard in glaucoma surgery [1-4]. Recently trabecular stents bypass the compromised trabecular meshwork, creating a direct route from the anterior chamber into Schlemm's canal and improving aqueous outflow [58]. In a prospective randomized clinical study, we investigated the long-term results, safety and efficacy of trabecular micro-bypass stent implantations as part of a routine cataract surgery in eyes with mild to moderate chronic open-angle glaucoma. In combined surgeries, phaco PC-IOL plus trabecular micro bypass implantation, has shown reasonable efficacy in lowering IOP and the necessary IOP reducing medications [9-11]. Our results showed a favourable benefit-to-risk profile.

There are currently three different modes of operation for MicroInvasive Glaucoma Surgery (MIGS): trabecular stents, suprachoroidal stents and subconjunctival stents [12-19]. Trabecular stents improve the outflow of aqueous humor into the Schlemm's canal. Examples are the iStent and the iStent inject $[20,21]$. Both stents are implanted while being guided by gonioscopy into Schlemm's canal. This is located at the level of the pigmented trabecular meshwork at the border to the non-pigmented trabecular meshwork. Suprachoroidal stents, such as the CyPass Micro-Stent (currently removed from the market), conduct the aqueous humor into the suprachoroidal space. The CyPass Micro-Stent is implanted more deeply, far below Schlemm's canal, into the supraciliary space. Stents for use in the subconjunctival space are, for example, the XEN gel implants, which are implanted far above Schlemm's canal, directly below the Schwalbe line, into the non-pigmented trabecular meshwork. The operating principle of subconjunctival outflow is like a small trabeculectomy.

iStent inject is a trabecular micro-bypass system with two preloaded implants made of heparin-coated, non-ferromagnetic titanium (Figure 1). The implants are very small. At $360 \mu \mathrm{m} \times 230 \mu \mathrm{m}$, they are the smallest medical devices used in humans. They have two ends; at one end, the inlet opening protrudes into the anterior chamber, while the other end opens out into the Schlemm's canal in a pointed conical shape with four lateral outlet openings. The middle part of the stent is fixed in the trabecular meshwork (Figure 2). The implants thus connect the anterior chamber with Schlemm's canal. They bridge the point of highest outflow resistance, the juxta-canalicular trabecular meshwork, the Fontana spaces, facilitating the outflow of aqueous humor there. The juxta-canalicular trabecular meshwork contains $50-70 \%$ of the total discharge resistance. Increased flow resistance in the trabecular meshwork is the primary cause of increased eye pressure in open angle glaucoma. The aqueous humor then flows from the anterior chamber into the stent, from the stent into Schlemm's canal, from Schlemm's canal into the collector channels, and from the collector channels into the episcleral veins. Two stents are always implanted, at 30 degrees, in the nasal lower quadrant, because the density of the discharging collector channels is highest there. The iStent inject received a CE mark in the EU and an Investigational Device Exemption (IDE) for clinical studies from the US FDA.

Implantation is possible under topical anaesthesia, and there is no need to stop anticoagulants and platelet aggregation inhibitors beforehand. In the combined procedure, following phacoemulsification and IOL implantation, the pupil can optionally be narrowed intracamerally with $1 \%$ acetylcholine chloride solution. To prepare for intraoperative gonioscopy, viscoelastic is injected into the anterior chamber, the operating microscope is tilted by 35 degrees and the patient's head is turned slightly to the contralateral side. The gonioscope, according to Jacobson, is placed on the cornea. To identify Schlemm's canal, we induce flow reversal from the episcleral vessels towards the

${ }^{\star}$ Correspondence to: Ulrich-Peter Best, Eye Clinic and Laser Eye Clinic Mainfranken, Am Oberen Marienbach 1, D-97421 Schweinfurt, Germany, E-Mail: dr.up.best@web.de

Received: April 01, 2019; Accepted: April 15, 2019; Published: April 18, 2019 


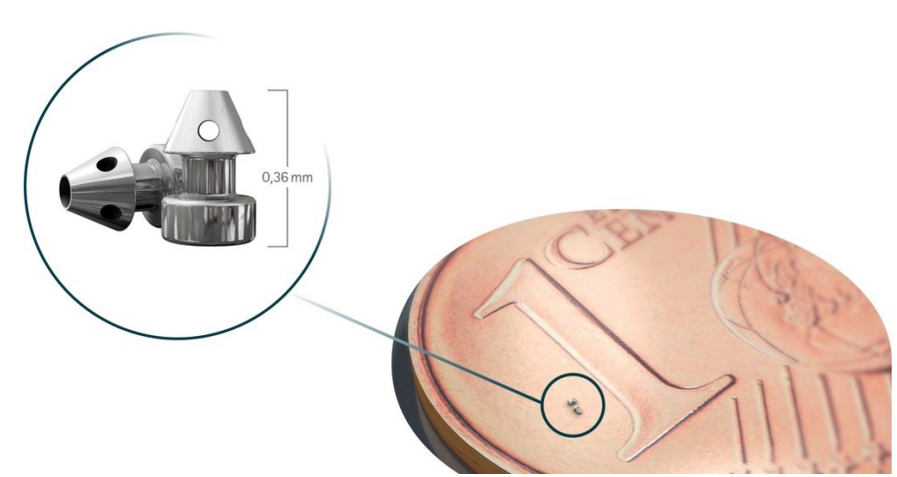

Figure 1. $\Delta$ iStent inject is a trabecular micro-bypass system with two preloaded implants made of heparin-coated, non-ferromagnetic titanium. The implants are very small. At 360 $\mu \mathrm{m} \times 230 \mu \mathrm{m}$, they are the smallest medical devices used in humans. They have two ends; at one end, the inlet opening protrudes into the anterior chamber, while the other end opens out into the Schlemm's canal in a pointed conical shape with four lateral outlet openings. The middle part of the stent is fixed in the trabecular meshwork. The implants connect the anterior chamber with Schlemm's canal. They bridge the point of highest outflow resistance, the juxta-canalicular trabecular meshwork, the Fontana spaces, facilitating the outflow of aqueous humor there. From the injector shown bottom left the two implants are released

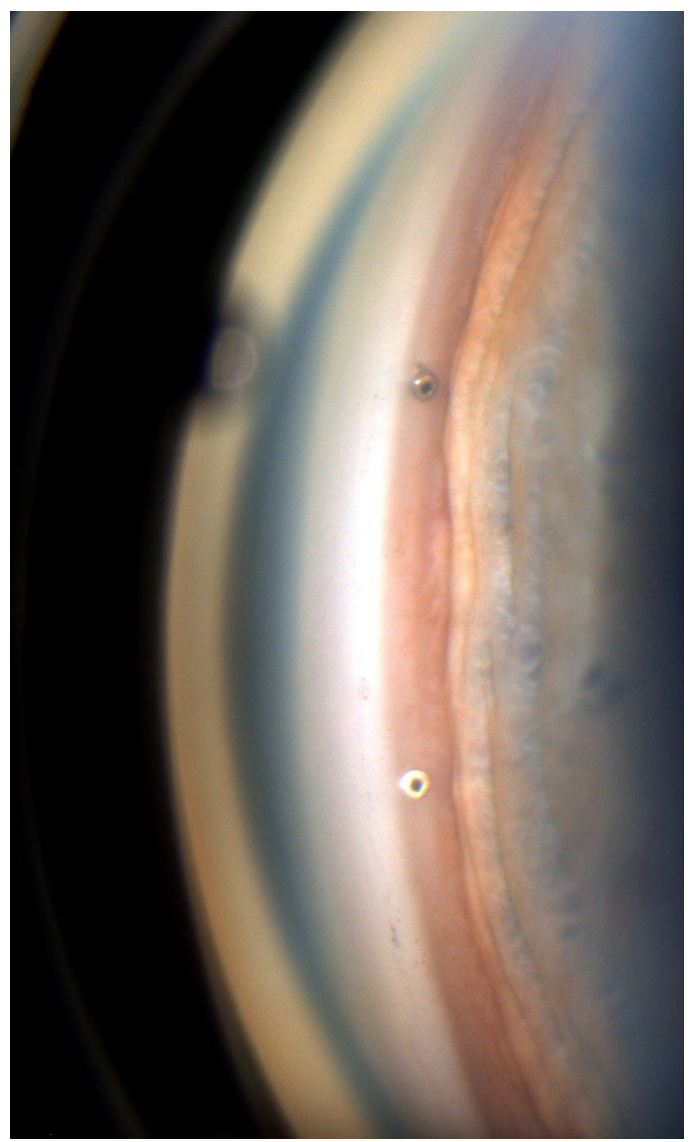

Figure 2. $\Delta$ Two in Schlemm's canal implanted iStents inject. The implantation is ab interno in between the pigmented and unpigmented trabecular meshwork, in the nasal inferior quadrant, where the collector channels density is highest

collector channels and Schlemm's canal prior to implantation. Flow reversal is achieved by gently pressing and massaging the sclera with hummingbird tweezers, for example. Schlemm's canal then turns slightly reddish for a few minutes as a result of the blood reflux. The injector has a trocar with a 23-gauge protective cover. This is inserted into the anterior chamber via a $1.4 \mathrm{~mm}$ wide clear corneal incision. The stent is then implanted ab interno into Schlemm's canal. Immediately after implantation, a small reflux haemorrhage occurs through the central opening of the implanted iStents inject, from the Schlemm's canal into the anterior chamber. This is a desired criterion of success for good positioning in the canal. Finally, it is possible to optionally introduce Trypan blue into the anterior chamber and document the blue coloration of the collector channels and episcleral veins.

In a controlled, randomized, single-blind study, we investigated the long-term results after trabecular micro-bypass stent implantation in routine cataract surgery in eyes with chronic open-angle glaucoma. The results are summarized in Table 1 . The average reduction in eye pressure with the combined procedure was $6.4 \mathrm{mmHg}$ (25.4\%). In the comparison group of standard cataract surgery alone without stent, eye pressure decreased by an average of $2.2 \mathrm{mmHg}(10.0 \%)$.

Standard cataract surgery alone also reduces eye pressure slightly $[22,23]$. Why? There are different theories about this. The natural crystalline lens becomes slightly larger and thicker with age. The anterior lens capsule shifts slightly farther forward. The crystalline lens exerts traction on the zonules. The traction force vector is directed in a radial (centripetal) and in an anterior direction. The traction is transmitted to the ciliary body and the trabecular meshwork. Traction increases with lens thickness and size. Traction probably compresses the drain channels, Schlemm's canal and the collector channels. Eye pressure increases slightly with age as a result of these anatomical changes. If the crystalline lens is later removed during cataract surgery, the radial tractions on the ciliary zonule disappear. Eye pressure then declines again to the previous level, at which the eye lens was even smaller and thinner.

The reduction in pressure due to cataract surgery is added to the reduction in pressure due to stent implantation. There is a direct additive effect, and the combined procedure proves to be a sensible combination here [24,25].

Glaucoma surgery is undergoing some changes. Micro-Invasive Glaucoma Surgery (MIGS) reduces eye pressure moderately. It is indicated for early and middle glaucoma stages, where eye pressure is moderately elevated, i.e., not above 30 under medication, with primary open-angle glaucoma, pseudo exfoliation glaucoma and pigment glaucoma. It facilitates early intervention in glaucoma progression and reduces drug exposure. The extent of the pressure reduction is comparable to that of two pressure-reducing drugs, falling between that of SLT and trabeculectomy. It avoids the possible complications of trabeculectomy. Pressure does not drop below 14 because we work against episcleral venous pressure [26].

Table 1. Phaco+HKL: IOP after standard cataract surgery in 35 eyes: the average intraocular pressure reduction was $2.5 \mathrm{mmHg}(11.4 \%)$ after three months, $2.1 \mathrm{mmHg}(9.5 \%)$ after six months, and $2.0 \mathrm{mmHg}(9.1 \%)$ after twelve months. Phaco + HKL + iStent ${ }^{\circledR}$ inject intraocular pressure after combined surgery in 36 eyes: the average intraocular pressure reduction was $6.9 \mathrm{mmHg}(27.5 \%)$ after three months, $6.5 \mathrm{mmHg}(25.9 \%)$ after six months, and $5.7 \mathrm{mmHg}(22.7 \%)$ after twelve months

\begin{tabular}{|c|c|c|c|c|c|}
\hline $\begin{array}{c}\text { Intraocular pressure } \\
\text { [mmHg] preoperative [0 } \\
\text { months] postoperative } \\
{[3-12 \text { months] }}\end{array}$ & 0 months & 3 months & 6 months & 9 months & 12 months \\
\hline Phaco+HKL $(\mathrm{n}=35)$ & 22 & 19.5 & 19.9 & 19.7 & 20 \\
\hline $\begin{array}{c}\text { Phaco+HKL+iStent }{ }^{\circledR} \\
\text { inject }(\mathrm{n}=36)\end{array}$ & 25.1 & 18.2 & 18.6 & 18.5 & 19.4 \\
\hline
\end{tabular}




\section{References}

1. Best UP, Domack H, Schmidt V (2005) Langzeitergebnisse nach Selektiver Lasertrabekuloplastik - eine klinische Studie an 269 Augen. Klin Monatsbl Augenheilkd 222: $326-331$

2. Best UP, Domack H, Schmidt V (2007) Augendrucksenkung nach Selektiver Lasertrabekuloplastik (SLT) mit zwei unterschiedlichen Lasersystemen und nach Argonlasertrabekuloplastik (ALT) - Eine kontrollierte prospektive klinische Studie an 284 Augen. Klin Monatsbl Augenheilkd 224: 173-179.

3. Landers J, Martin K, Sarkies N (2012) A twenty-year follow-up study of trabeculectomy: risk factors and outcomes. Ophthalmology 119: 694-702. [Crossref]

4. Razeghinejad MR, Spaeth GL (2011) A history of the surgical management of glaucoma. Optom Vis Sci 88: E39-47.

5. Fea AM, Belda JI, Rękas M, Jünemann A, Chang L, et al. (2014) Prospective unmasked randomized evaluation of the iStent inject $($ versus two ocular hypotensive agents in patients with primary open-angle glaucoma. Clin Ophthalmol 8: 875-882. [Crossref]

6. Hunter KS, Fjield T, Heitzmann H, Shandas R, Kahook MY (2014) Characterization of micro-invasive trabecular bypass stents by ex vivo perfusion and computational flow modeling. Clin Ophthalmol 8: 499-506. [Crossref]

7. Irshad FA, Mayfield MS, Zurakowski D, Ayyala RS (2010) Variation in Schlemm's canal diameter and location by ultrasound biomicroscopy. Ophthalmology 117: 916920 .

8. Lindstrom R, Lewis R, Hornbeak DM, Voskanyan L, Giamporcaro JE, et al. (2016) Outcomes Following Implantation of Two Second-Generation Trabecular MicroBypass Stents in Patients with Open-Angle Glaucoma on One Medication: 18-Month Follow-Up. Adv Ther 33: 2082-2090.

9. Arriola-Villalobos P, Martínez-de-la-Casa JM, Díaz-Valle D, García-Vidal SE, Fernández-Pérez C, et al. (2013) Mid-term evaluation of the new Glaukos iStent with phacoemulsification in coexistent open-angle glaucoma or ocular hypertension and cataract. Br J Ophthalmol 97: 1250-1255. [Crossref]

10. Craven ER, Katz LJ, Wells JM, Giamporcaro JE (2012) Cataract surgery with trabecular micro-bypass stent implantation in patients with mild-to-moderate openangle glaucoma and cataract: two-year follow-up. J Cataract Refract Surg 38: 13391345. [Crossref]

11. Poley BJ, Lindstrom RL, Samuelson TW, Schulze R (2009) Intraocular pressure reduction after phacoemulsification with intraocular lens implantation in glaucomatous and nonglaucomatous eyes: evaluation of a causal relationship between the natural lens and open-angle glaucoma. J Cataract Refract Surg 35: 1946-1955. [Crossref]

12. Brandão LM, Grieshaber MC (2013) Update on minimally invasive glaucoma surgery (MIGS) and new implants. J Ophthalmol 2013: 705915. [Crossref]
13. Saheb H, Ahmed II (2012) Micro-invasive glaucoma surgery: current perspectives and future directions. Curr Opin Ophthalmol 23:96-104. [Crossref]

14. Spiegel D, Kobuch K, Hill RA, Gross RL (1999) Schlemm's canal implant: a new method to lower intraocular pressure in patients with POAG? Ophthalmic Surg Lasers 30: 492-494.

15. Spiegel D, Kobuch K (2002) Trabecular meshwork bypass tube shunt: initial case series. Br J Ophthalmol 86: 1228-1231.

16. Stalmans I (2016) A Minimally Invasive Approach to Sub-conjunctival Outflow: 1 Year Results of an Ab-interno Gelatin Stent for the Treatment of Primary Open Angle Glaucoma. International Congress of Glaucoma Surgery poster. Scandinavica Foundation.

17. Vold S, Ahmed IIK, Craven ER, Mattox C, Stamper R, et al (2016) Two-Yea COMPASS Trial Results: Supraciliary Microstenting with Phacoemulsification in Patients with Open-Angle Glaucoma and Cataracts for the CyPass Study Group. Ophthalmology 123: 10.

18. Voskanyan L, García-Feijoó J, Belda JI (2014) Prospective, unmasked evaluation of the iStent ${ }^{\circledR}$ inject system for open-angle glaucoma: synergy trial. Adv Ther 31: 189201. [Crossref]

19. Vold SD, Voskanyan L, Tetz M (2016) Newly diagnosed primary open-angle glaucoma randomized to 2 trabecular bypass stents or prostaglandin: outcomes through 36 months. Ophthalmol Ther 5: 161-172. [Crossref]

20. Bahler CK, Hann CR, Fjield T (2012) Second-generation trabecular meshwork bypass stent (iStent inject) increases outflow facility in cultured human anterior segments. $\mathrm{Am}$ J Ophthal 153: 1206-1213. [Crossref]

21. Bahler CK, Smedley GT, Zhou J, Johnson DH (2004) Trabecular bypass stent decrease intraocular pressure in cultured human anterior segments. Am J Ophthal 138: 988-994. [Crossref]

22. Berdahl JP (2009) Cataract Surgery to Lower Intraocular Pressure. Middle East Afr J Ophthalmol 16: 119-122.

23. Slabaugh MA, Bojikian KD, Moore DB, Chen PP (2014) The Effect of Phacoemulsification on Intraocular Pressure in medically controlled Open-Angle Glaucoma Patients. Am J Ophthalmol 157: 26-31. [Crossref]

24. Katz LJ, Erb C, Guillamet AC, Fea AM, Voskanyan L, et al. (2015) Prospective, randomized study of one, two, or three trabecular bypass stents in open angle glaucoma subjects on topical hypotensive medication. Clin Ophthalmol 9: 2313-2320. [Crossref]

25. Klamann MK, Gonnermann J, Pahlitzsch M, Maier AK, Joussen AM, et al. (2015) iStent inject in phakic open angle glaucoma. Graefes Arch Clin Exp Ophthalmol 253: 941-947.

26. Shrivastava A, Singh K (2010) The effect of cataract extraction on intraocular pressure. Curr Opin Ophthalmol 21: 118-122.

Copyright: (C2019 Best UP. This is an open-access article distributed under the terms of the Creative Commons Attribution License, which permits unrestricted use, distribution, and reproduction in any medium, provided the original author and source are credited. 